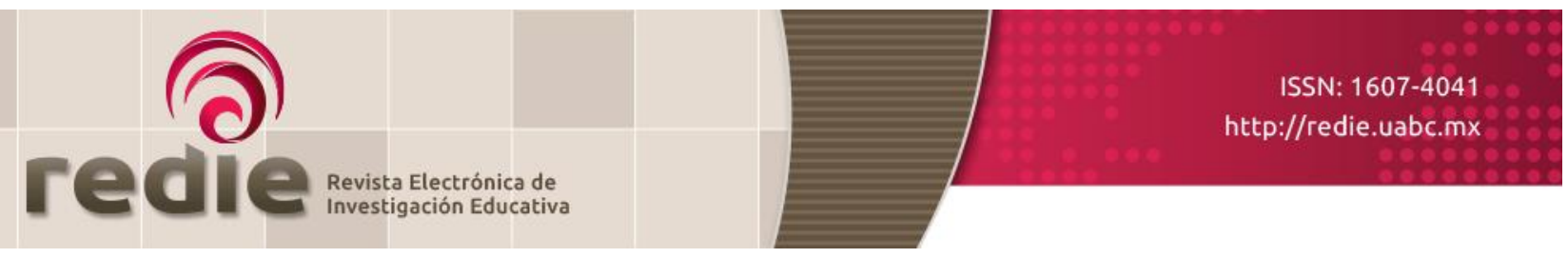

Vol. 22, 2020/e03

\title{
Bienestar social en la etapa universitaria según factores sociodemográficos en estudiantes de Educación
}

\section{Social Well-Being in College According to Sociodemographic Factors in Education Students}

\author{
Ramón Chacón-Cuberos (*) rchacon@ugr.es \\ Asunción Martínez-Martínez (*) asuncionmm@ugr.es \\ Pilar Puertas-Molero (*) pilarpuertas@correo.ugr.es \\ Virginia Viciana-Garófano (*) vviciana@ugr.es \\ Gabriel González-Valero (*) gabri1322@correo.ugr.es \\ Félix Zurita-Ortega (*) felixzo@ugr.es \\ (*) Universidad de Granada \\ (Recibido: 8 de enero de 2018; Aceptado para su publicación: 12 de abril de 2018)
}

Cómo citar: Chacón-Cuberos, R., Martínez-Martínez, A., Puertas-Molero, P., Viciana-Garófano, V., González-Valero, G. y Zurita-Ortega, F. (2020). Bienestar social en la etapa universitaria según factores sociodemográficos en estudiantes de educación. Revista Electrónica de Investigación Educativa, 22, e03, 1-9. https://doi.org/10.24320/redie.2020.22.e03.2184

\section{Resumen}

El propósito de este estudio fue analizar el bienestar social percibido de estudiantes universitarios de Ciencias de la Educación en función de factores de tipo sociodemográfico. Se realizó un estudio no experimental, ex post facto, de carácter descriptivo mediante una medición única. La muestra estuvo constituida por 313 estudiantes universitarios de diversos grados de Educación. Los resultados indican que las mujeres muestran mayores niveles de contribución y coherencia social, mientras que los estudiantes (hombres y mujeres) que acceden a la universidad mediante formación profesional u otra vía se perciben con mayor bienestar social. Se demuestra que la integración social es mayor en las zonas más urbanizadas y que aquellos universitarios que conviven con otros presentan más aceptación social. Asimismo, vivir en zonas rurales se asoció a una mayor actualización y coherencia social por la mayor facilidad de entender la dinámica social. La edad correlacionó negativamente con la coherencia social.

Palabras clave: Estudiantes universitarios, bienestar social, condiciones de vida, estilo de vida, género.

\section{Abstract}

This study sought to examine the perceived social well-being of students of educational sciences, based on sociodemographic factors. A non-experimental, ex-post-facto descriptive study was performed by way of a single measurement. The sample comprised 313 college students from different education degrees. The results show that women exhibit greater levels of social coherence and contribution, while students (men and women) that enter university through vocational training or other non-high school routes have greater perceived well-being. It was shown that social integration is greater in more urbanized areas and college students that live with other students display greater social acceptance. In addition, living in rural 
areas was associated with greater social actualization and coherence as social dynamics are more easily understood. Age correlated negatively with social coherence.

Keywords: University students, well-being, living condition, life style, gender.

\section{Introducción}

El período universitario viene marcado por el inicio de los estudios superiores para la obtención de la formación profesionalizante que permite la inserción laboral en la adultez joven (Haapanen y Tervo, 2012). Esta etapa supone diversidad de cambios en el estilo de vida e incluye la configuración de relaciones sociales novedosas, la emancipación familiar, inmersión en entramados académicos diferentes o tener que vivir de modo autosuficiente (Chacón et al., 2017). Asimismo, el fin de la adolescencia supone la conclusión de una etapa en la que el adulto joven ha terminado de explorar su identidad y comienza a afianzarla, pudiendo exhibir aún comportamientos inestables (Haapanen y Tervo, 2012). En este sentido González-Peiteado et al. (2017) establecen que la percepción de los estudiantes universitarios sobre su calidad de vida resulta un elemento sustancial en la configuración de su bienestar subjetivo, el cual resulta esencial en la configuración de su personalidad.

El concepto de bienestar social fue iniciado por Keyes y Shapiro (1998) y posteriormente fue delimitado por otros autores. Al inicio el estudio del bienestar se desprendía del constructo bienestar subjetivo, el cual comprende tres dimensiones básicas, como son la satisfacción con la vida, la felicidad y afectos negativos y positivos derivados de la existencia del ser humano (Blanco y Díaz, 2005; Zubieta y Delfino, 2010). En este sentido, el concepto de bienestar se ha configurado a raíz de cómputo conformado por las oportunidades vitales, el transcurso de los hechos de su vida y las experiencias emocionales que las comprenden (Aknin et al., 2013; Blanco y Díaz, 2005; Oberle et al., 2010).

Como concepción más reciente, el bienestar psicológico hace referencia a la capacidad de esfuerzo para afrontar nuevos retos, el desarrollo personal y la iniciativa para la consecución de logros (Chavarría y Barra, 2014). En este sentido, y partiendo de la estructura social en la que nos movemos, no cabe duda de que el concepto de bienestar se vincula con las relaciones interpersonales y sociales, así como con los recursos y el funcionamiento y contribución social (Burke et al., 2010). Estudios como el de Aknin et al. (2013) o Blanco y Díaz (2005) configuran el concepto bienestar social, el cual se basa en el significado que se le otorga a las circunstancias vivenciales de cada individuo en la sociedad, así como al funcionamiento del mismo dentro de ella, configurando cinco grandes dimensiones: integración social, referida a la calidad de las relaciones sociales; aceptación social, asociada al sentimiento de pertenencia a grupos sociales establecidos; contribución social, dimensión que concreta la capacidad del sujeto para la organización y realización de acciones con objetivos sociales; actualización social, referida al dinamismo que presenta la sociedad y sus instituciones y la confianza depositada en las mismas; y coherencia social, relacionada con la capacidad de entender la dinámica social, así como la preocupación por comprender lo que sucede en la sociedad (Blanco y Díaz, 2005; Ding et al., 2017).

En este sentido, Zubieta y Delfino (2010) hacen especial énfasis en la conceptualización del término desde una perspectiva psicosocial que se aleja de emociones negativas como el estrés, la ansiedad o la depresión, muchas de las cuales redundan en la población universitaria dado el estrés académico generado y las características propias de la etapa, como menor debilidad en las relaciones familiares o desarrollo de hábitos no saludables que podría alejarse del concepto de bienestar (Cotton et al., 2002; Ding et al., 2017). Por estos motivos, resulta de interés su estudio en este sector, dado que elevados niveles de satisfacción con la vida y bienestar se asociarán con emociones positivas como altos niveles de autoestima, autoaceptación o relaciones sociales positivas con pares (Diener et al., 2010). Asimismo, estos factores se relacionarán a un mejor rendimiento académico en dicha etapa educativa, hábitos saludables que evitarán la presencia de patologías futuras, así como mayores niveles de participación y compresión social (Chacón et al., 2017).

Varios trabajos de investigación han estudiado las asociaciones existentes entre el bienestar social y otros factores psicológicos o sociodemográficos, como son el género, la edad o lugar de residencia (Ding et al., 
2017; Friedman, 2011; Rollero y De Piccoli, 2010). Salanova et al. (2005) revelan cómo el bienestar psicológico de estudiantes universitarios se relaciona con aspectos psicosociales como es el burnout estado de estrés y fatiga emocional y física debida a factores laborales- o el engagement -concepto que alude a un estado cognitivo positivo para el trabajo- hallando asociaciones con los niveles de felicidad global, compromiso, autoeficacia y satisfacción con la vida. Asimismo, un estudio reciente realizado por Burke et al. (2010) revela la importancia de las relaciones sociales en el bienestar social, de tal forma que las redes sociales pueden generar niveles elevados de autoestima y satisfacción con la vida en el nivel universitario, lo que constituye un componente esencial para el bienestar.

En relación con los aspectos sociodemográficos, Rollero y De Piccoli (2010) muestran que las relaciones sociales y el ambiente contextual de la persona representan factores clave para entender el nivel de calidad de vida y el bienestar percibido, los cuales se ven influenciados por las características del medio ambiente (Oberle et al., 2010). De hecho, se puede establecer que tener una percepción positiva del lugar de residencia puede constituir un predictor eficaz del bienestar en poblaciones concretas, incluso en estudiantes universitarios, a través de las emociones, creencias y comportamientos que implican (Rollero y De Piccoli, 2010). Por otro lado, Ding et al. (2017) hacen hincapié en la relación existente entre los roles de género y el bienestar percibido, reflejado en un menor bienestar en el caso del género femenino en estudiantes universitarios de origen asiático.

Con base en lo expuesto, se identifica la existencia de variaciones en el bienestar social percibido de estudiantes universitarios según factores sociodemográficos, siendo un campo de interés dada la escasa presencia de estudios que incluyan estos factores de forma conjunta y la compleja configuración psicosocial del adulto joven en esta etapa, a la cual se le unen presiones académicas que generan estados de estrés y ansiedad. Así, este estudio busca definir los niveles de bienestar social en sus cinco dimensiones en una población universitaria de Ciencias de la Educación, así como analizar las relaciones existentes con base en el género, edad, lugar y zona de residencia, y vía de acceso a la universidad.

\section{Método}

Se realizó un estudio cuantitativo, no experimental, ex post facto, de carácter descriptivo realizado mediante una única medición de grupo. La muestra estuvo constituida por 313 estudiantes universitarios con una edad media de 21.26 años (DT = 3.98); el 19.6\% ( $n=53$ ) fueron hombres y un $83.1 \%(n=260)$ mujeres. Se realizó un muestreo no probabilístico seleccionando a los participantes por conveniencia.

Se utilizaron dos instrumentos:

Escala de Bienestar Social, extraída del instrumento original de Keyes y Shapiro (1998) y validada al español por Blanco y Díaz (2005). El test se compone de 33 ítems, los cuales se puntúan mediante una escala tipo Likert de cinco opciones, donde 1 = Totalmente en desacuerdo y 5 = Totalmente de Acuerdo. Los ítems se estructuraron en cinco dimensiones y se distribuyeron los ítems de siete en siete. Los siete primeros corresponden a Integración social, los siete siguientes a Aceptación social, siguiendo con Contribución social, Actualización social y Coherencia Social. Se obtuvo una fiabilidad a = .851 para este instrumento, la cual resulta aceptable. El análisis de la consistencia interna por dimensiones reflejó los siguientes valores: Dimensión 1 Integración social $(a=.700)$; Dimensión 2 Aceptación social $(a=.793)$; Dimensión 3 Contribución social $(a=.816)$; Dimensión 4 Actualización social $(a=.677)$; Dimensión 5 Coherencia social $(a=.669)$.

Cuestionario ad hoc. Se elaboró un cuestionario específico para el registro de las variables de tipo sociodemográfico. Se consideró género (categorizado en masculino y femenino), edad, población (tipo de población en la que reside cada participante, codificada en urbana (>15000 habitantes), semiurbana (entre 5000 y 15000 habitantes) y rural (<5000 habitantes), lugar de residencia (tipo residencia de los participantes durante el curso académico, considerando piso compartido, hogar familiar y residencia universitaria), y vía de acceso (determina la vía de acceso a la universidad, considerado bachillerato, formación profesional u otras). 
Se solicitó la colaboración de los participantes, todos estudiantes de Ciencias de la Educación en la Universidad de Granada, a través de una carta en la cual se detallaba la naturaleza del estudio con el fin de obtener su consentimiento informado. La recogida de los datos se realizó en horario lectivo. El proceso transcurrió sin incidencias, siempre con la presencia de los investigadores para que se realizase una correcta aplicación de los instrumentos descritos, así como para la resolución de dudas. Cabe señalar que se ha respetado el derecho de confidencialidad de todos los participantes y que se invalidó un total de 14 cuestionarios por estar mal cumplimentados.

Se utilizó el programa SPSS 22.0 para la realización del análisis estadístico y se utilizaron frecuencias y medias para la realización de los descriptivos básicos. Para estudiar las relaciones de las variables se empleó la comparación de medias mediante $t$ de Student o Anova de un factor dependiendo de la naturaleza de las variables. Para determinar la fiabilidad interna del instrumento se utilizó el coeficiente alfa de Cronbach, determinando el Índice de confiabilidad en 95.5\%.

\section{Resultados}

La tabla I revela los descriptivos básicos del estudio realizado. La muestra estuvo representada en un 83.1\% ( $n=260)$ por mujeres y en un 16.9\% ( $n=53$ ) por hombres. La distribución según lugar de residencia revela que un 13.7\% $(n=43)$ residía en zonas rurales, que un $17.9 \%(n=56)$ lo hacía en zonas semiurbanas y que un $68.4 \%$ ( $n=214$ ) residía zonas urbanas. Asimismo, los resultados desprenden que un $69.6 \%$ de los participantes vivía en piso compartido, un $28.1 \%$ lo hacía en el domicilio familiar y un $2.2 \%$ vivía en residencia universitaria. Sobre la vía de acceso a la universidad, se obtuvo que un $47.9 \%$ había accedido a la titulación a través de bachillerato, un 49.8\% accedió mediante formación profesional y un $2.2 \%$ lo hizo mediante otra vía. En relación con el bienestar social, se obtienen las puntuaciones medias para cada una de las dimensiones en la muestra de estudio; Integración social (3.73 \pm 0.52$)$, Aceptación social (3.16 \pm 0.53$)$, Contribución social $(4.13 \pm 0.62)$, Actualización social $(3.34 \pm 0.57)$ y Coherencia social $(3.53 \pm 0.53)$.

Tabla I. Descriptivos básicos

\begin{tabular}{l|l|l}
\hline \multirow{2}{*}{ Género } & Masculino & $16.9 \%(n=53)$ \\
& Femenino & $83.1 \%(n=260)$ \\
\hline \multirow{3}{*}{ Población } & Rural & $13.7 \%(n=43)$ \\
& Semiurbano & $17.9 \%(n=56)$ \\
& Urbano & $68.4 \%(n=214$ \\
\hline \multirow{2}{*}{$\begin{array}{l}\text { Lugar de } \\
\text { residencia }\end{array}$} & Piso compartido & $69.6 \%(n=218)$ \\
& Hogar familiar & $28.1 \%(n=88$ \\
& Residencia & $2.2 \%(n=7)$ \\
\hline \multirow{2}{*}{$\begin{array}{l}\text { Acceso a la } \\
\text { universidad }\end{array}$} & Bachillerato & $47.9 \%(n=150)$ \\
\cline { 2 - 3 } & FP & $49.8 \%(n=156)$ \\
\hline \multirow{4}{*}{$\begin{array}{l}\text { Bienestar } \\
\text { social }\end{array}$} & Otra & $2.2 \%(n=7)$ \\
\cline { 2 - 3 } & Aceptación & $3.73 \pm .52$ \\
& Contribución & $4.16 \pm .53$ \\
\cline { 2 - 3 } & Actualización & $3.34 \pm .52$ \\
\hline
\end{tabular}

La tabla II revela las relaciones existentes entre el género de los participantes y el bienestar social de los mismos, obteniendo diferencias estadísticamente significativas para la Contribución social $(p=0.023)$ y la Coherencia social $(p=0.035)$. Para la primera dimensión, las mujeres obtienen puntuaciones medias más elevadas que los hombres (4.16 versus 3.95). En una línea similar, las mujeres muestran valores más elevados que los hombres en la Coherencia social (3.56 versus 3.39). Igualmente, se muestran las asociaciones existentes entre bienestar social y vía de acceso a la universidad, concretando diferencias estadísticamente significativas para la Aceptación social $(p=0.027)$ y la Coherencia social $(p=0.005)$. Para la primera dimensión, son aquellos participantes que acceden mediante otra vía los que se adhieren a una 
media más elevada en comparación con los que acceden mediante formación profesional o bachillerato sucesivamente (3.51 versus 3.21 versus 3.09). En relación con la Coherencia social, son los estudiantes que accedieron mediante formación profesional los que muestran niveles más elevadas en comparación con bachillerato u otra vía (3.63 versus 3.44 versus 3.42).

Tabla II. Bienestar social según género y vía de acceso a la universidad

\begin{tabular}{l|c|c|c|c|c|c|c}
\hline & \multicolumn{3}{|c|}{ Género } & \multicolumn{3}{c}{ Acceso a universidad } \\
\hline & Masculino & Femenino & $p$ & Bachiller & FP & Otra & $p$ \\
\hline Integración social & $3.84 \pm 0.41$ & $3.71 \pm 0.53$ & 0.097 & $3,71 \pm 0.46$ & $3.74 \pm 0.57$ & $3.67 \pm 0.55$ & 0.835 \\
Aceptación social & $3.05 \pm 0.56$ & $3.18 \pm 0.52$ & 0.105 & $3.09 \pm 0.50$ & $3.21 \pm 0.55$ & $3.51 \pm 0.51$ & $0.027^{*}$ \\
Contribución social & $3.95 \pm 0.50$ & $4.16 \pm 0.63$ & $0.023^{*}$ & $4.07 \pm 0.59$ & $4.19 \pm 0.65$ & $3.97 \pm 0.24$ & 0.213 \\
Actualización social & $3.30 \pm 0.50$ & $3.35 \pm 0.58$ & 0.543 & $3.40 \pm 0.55$ & $3.29 \pm 0.59$ & $3.47 \pm 0.17$ & 0.214 \\
Coherencia social & $3.39 \pm 0.51$ & $3.56 \pm 0.52$ & $0.035^{*}$ & $3.44 \pm 0.48$ & $3.63 \pm 0.56$ & $3.42 \pm 0.08$ & $0.005^{* *}$ \\
\hline
\end{tabular}

Notas: $p<0.05^{*} ; p<0.01^{* *} ; p<0.001 * * *$

FP: Formación Profesional

La relación existente entre las dimensiones de bienestar social y el lugar y población de residencia de residencia queda reflejada en la tabla III. Se revelan diferencias estadísticamente significativas para la Aceptación social $(p=0.007)$, la Actualización social $(p=0.013)$ y la Coherencia social $(p=0.034)$. Para la Aceptación social, los universitarios que viven en residencia universitaria muestran puntuaciones medias más elevadas que los que lo hacen en hogar familiar y piso compartido (3.66 versus 3.06 versus 3.19). Se observa la misma tendencia en el caso de la Actualización social (3.88 versus 3.25 versus 3.36) y la Coherencia social (4.02 versus 3.48 versus 3.54). En relación con el tipo de población, se observan diferencias estadísticamente significativas para la Integración social $(p=0.019)$, la Actualización social $(p=$ $0.001)$ y la Coherencia social $(p=0.010)$. Los mayores niveles de Integración social se muestran en zonas urbanas con respecto a las semiurbanas y las rurales (3.78 versus. 3.59 versus 3.63). En el caso de la Actualización social, las mayores medias se adhieren a las zonas rurales, seguidas de las semiurbanas y las urbanas (3.62 versus 3.37 versus 3.28). Finalmente, se observa la misma tendencia en el caso de la Coherencia social, revelando las zonas rurales las puntuaciones más elevadas (3.71 versus 3.63 versus 3.47).

Tabla III. Bienestar social según lugar y población de residencia

\begin{tabular}{l|c|c|c|l|l|l|l|c}
\hline & \multicolumn{3}{|c|}{ Lugar de residencia } & \multicolumn{1}{c}{ Población } \\
\hline & PC & HF & RU & $p$ & \multicolumn{1}{c}{ Rural } & SU & Urbano & $p$ \\
\hline Integración s. & $3.76 \pm 0.52$ & $3.64 \pm 0.52$ & $3.93 \pm 0.38$ & 0.110 & $3.63 \pm 0.57$ & $3.59 \pm 0.57$ & $3.78 \pm 0.48$ & $0.019^{*}$ \\
Aceptación s. & $3.19 \pm 0.53$ & $3.06 \pm 0.51$ & $3.66 \pm 0.25$ & $0.007^{* *}$ & $3.15 \pm 0.47$ & $3.29 \pm 0.60$ & $3.13 \pm 0.52$ & 0.152 \\
Contribución s. & $4.08 \pm 0.59$ & $4.21 \pm 0.68$ & $4.52 \pm 0.32$ & 0.064 & $4.25 \pm 0.50$ & $4.17 \pm 0.60$ & $4.09 \pm 0.64$ & 0.250 \\
Actualización s. & $3.36 \pm 0.49$ & $3.25 \pm 0.70$ & $3.88 \pm 0.58$ & $0.013^{*}$ & $3.62 \pm 0.31$ & $3.37 \pm 0.60$ & $3.28 \pm 0.58$ & $0.001^{* * *}$ \\
Coherencia s. & $3.54 \pm 0.51$ & $3.48 \pm 0.54$ & $4.02 \pm 0.57$ & $0.034^{*}$ & $3.71 \pm 0.50$ & $3.63 \pm 0.48$ & $3.47 \pm 0.53$ & $0.01^{* *}$ \\
\hline
\end{tabular}

Notas: $p<0.05^{*} ; p<0.01^{* *} ; p<0.001^{* * *}$

PC: Piso Compartido; HF: Hogar Familiar; RU: Residencia Universitaria; SU: Semi-Urbano

Por último, se realizaron correlaciones bivariadas entre todas las dimensiones del bienestar social y la edad de los participantes (ver tabla IV). Se obtuvieron diferencias estadísticamente significativas a nivel $p$ $<0.01$ entre todas las dimensiones, revelando relaciones directas y positivas. Las relaciones de mayor fortaleza fueran la establecida entre la Actualización social y la Aceptación social $(r=0.400)$ y la dada entre la Coherencia social y la Contribución social $(r=0.416)$. Por otro lado, la edad se relacionó únicamente con la Coherencia social $(p<0.001)$, revelando una relación indirecta o negativa $(r=-0.159)$. 
Tabla IV. Correlaciones bivariadas de las dimensiones de bienestar social y la edad

\begin{tabular}{l|c|c|c|c|c}
\hline & Integración & Aceptación & Contribución & Actualización & Coherencia \\
\hline Edad & -0.089 & -0.074 & -0.062 & -0.104 & $-0.159^{* *}$ \\
Integración & & $0.264^{* *}$ & $0.536^{* *}$ & $0.268^{* *}$ & $0.215^{* *}$ \\
Aceptación & & & $0.293^{* *}$ & $0.400^{* *}$ & $0.246^{* *}$ \\
Contribución & & & & $0.345^{* *}$ & $0.416^{* *}$ \\
Actualización & & & & & $0.197^{* *}$ \\
\hline
\end{tabular}

Notas: ** La correlación es significativa al nivel 0.01 (bilateral).

\section{Discusión}

El bienestar subjetivo y social en estudiantes universitarios constituye una realidad que exige ser estudiada, ya que esta etapa puede revestir cierta complejidad en relación con factores psicosociales concretos -síndrome de burnout, bajo autoconcepto y autoestima, consumo de sustancias nocivas, etc.debido al estrés académico, la emancipación familiar y la configuración de nuevas relaciones sociales. Ante esta perspectiva, este estudio pretende analizar los niveles de bienestar social y sus dimensiones en una muestra de estudiantes universitarios de Ciencias de la Educación en función de factores sociodemográficos, siguiendo una línea similar a otros trabajos de investigación, como los realizados por Cookingham y Ryan (2015), Fernández y Gutiérrez (2013), Lalive y Stutzer (2010), Rollero y De Piccoli (2010) y Zubieta y Delfino (2010), entre otros.

El estudio del bienestar social en función del género reveló que las mujeres mostraban mayores niveles de Contribución social y de Coherencia social. Estos resultados pueden justificarse por las premisas dadas por Ding et al. (2017), quienes hacen hincapié en la relación existente entre los roles de género y el bienestar percibido, dado que obtuvieron un menor bienestar en el caso de las mujeres de origen asiático que cursaban estudios universitarios. Estos hallazgos pueden parecer contradictorios, sin embargo, dichos autores atribuyen los resultados a una mayor conciencia por parte de las mujeres sobre los procesos interpersonales y sociales, lo cual podría justificar que el género femenino posea una mayor capacidad para realizar acciones con objetivos sociales (Ding et al., 2017; Perry-Parrish y Zeman, 2011).

En una línea similar, tanto Lalive y Stutzer (2010) como Zubieta y Delfino (2010) encontraron que las mujeres poseían mejores relaciones sociales con el entorno que los hombres, se sentían más útiles y confiaban en mayor medida en el futuro de la sociedad. Dichos resultados van en consonancia con los obtenidos en este estudio, dado que los estudiantes universitarios de género femenino obtuvieron mayores puntuaciones en Contribución y Coherencia social, dimensiones relacionadas con la capacidad de comprender y participar en dinámicas sociales. Esto es debido a que las mujeres pueden presentar un locus interno más elevado, así como mayor autoconfianza y motivación para participar socialmente (PerryParrish y Zeman, 2011; Zubieta y Delfino, 2010).

Resulta de interés destacar también que aquellos estudiantes que accedieron al grado universitario por vías diferentes al bachillerato mostraban niveles más elevados de Aceptación y Coherencia social, se evidencia que cursar formación profesional u otras titulaciones de forma previa incrementa el sentimiento de pertenencia a la sociedad y amplía la capacidad para comprenderla, especialmente por el dinamismo que implica, el desarrollo de competencias formativas y laborales y la configuración de relaciones sociales interculturales dentro del ámbito universitario (Lorenzo et al., 2014). En esta línea, Martínez-Martínez et al. (2016) muestran cómo acceder a la universidad mediante ciclos formativos otorga un mejor proceso de adaptación por la adecuación del itinerario académico, facilitando el tránsito entre etapas. En este sentido, aquellos jóvenes presentarán menores niveles de estrés y mayor satisfacción hacia la titulación, representando un factor esencial sobre el bienestar psicológico y subjetivo, de tal forma que la calidad de vida universitaria estará en íntima relación con el bienestar social (González-Peiteado et al., 2017).

El estudio del bienestar social en relación con el lugar de residencia revela que los universitarios que residen en zonas urbanas presentan mayores niveles de Integración social, mientras que los que viven en 
zonas rurales durante el curso académico poseen niveles más elevados en Coherencia y Actualización social, dimensiones que se refieren a la confianza depositada en la sociedad y la capacidad para entender su dinámica; estos hallazgos son similares a lo encontrado por Rollero y De Piccoli (2010) en una muestra de estudiantes universitarios italianos de primer año, dichos autores concluyen que vivir en zonas urbanas se relaciona con una percepción más globalizada de bienestar, mientras que residir en territorios más pequeños está asociado con lazos afectivos más fuertes (López y Wodtke, 2010; Oishi, 2010). En efecto, residir en zonas rurales se asocia con menores niveles de estrés en la rutina diaria, menor presión social o mayores niveles de relajación, factores que se asocian a patrones físicos y psicológicos relacionados con mayores niveles de bienestar (Oswald et al., 2011;). Del mismo modo, resulta evidente que residir en zonas urbanas se asocie a mayores niveles de Integración social, dado que éstas gozan de una mayor interculturalidad a nivel académico, social y laboral (Haapanen y Tervo, 2012; Oishi, 2010).

Estudios como el de González-Peiteado et al. (2017) muestran cómo la percepción de las construcciones cognitivas realizadas por los estudiantes varía según su edad. En el presente estudio se observó que la edad correlacionaba de forma inversa con la Coherencia social, dimensión que se refiere a la capacidad para entender la dinámica social. En este sentido, parece ser que al aumentar la edad disminuye la compresión de las fluctuaciones que se producen en las relaciones personales a nivel universitario, así como del entramado social (Aknin et al., 2013; Yu et al., 2010). Justificando estos hallazgos con base en las relaciones sociales definidas, Burke et al. (2010) señalan que el establecimiento de relaciones de amistad muestra una relación inversa con la edad, justificando estos resultados por la configuración del seno familiar. Asimismo, su justificación puede hallarse en la mayor importancia que se le da al bienestar personal y familiar a medida que aumenta la edad, en detrimento del activismo social (Luong et al.,2011).

Algunas limitaciones a considerar en este estudio son: el diseño de la investigación (tipo descriptivo y corte transversal) que no establece relaciones causales -aunque destaca por su facilidad de ejecución y capacidad para definir el estado de una cuestión-; la selección de la muestra, que comprende únicamente estudiantes de Ciencias de la Educación sin considerar otras titulaciones, además de emplear un muestreo no probabilístico, y por último, habría sido interesante incluir otros factores psicosociales relacionados con la satisfacción con la vida, autoconcepto o autoestima, que guardan una estrecha relación con el bienestar social percibido. En este sentido, se establecen como perspectivas futuras tanto la inclusión de otras variables de tipo psicosocial como la ampliación de la muestra a estudiantes de otras áreas, con el fin de proporcionar una descripción más amplia y exhaustiva de los niveles de bienestar social en la población universitaria.

Como principales conclusiones, se demuestra que las mujeres poseen mayores niveles de Contribución y Coherencia social debido al diferente valor que otorga al desarrollo de relaciones interpersonales, basado en un componente altruista. En esta línea, los estudiantes que acceden a la universidad mediante formación profesional u otra vía se perciben con mayor bienestar social, principalmente debido a las competencias formativas y relaciones sociales configuradas en tramos educativos anteriores. Se demuestra que la Integración social es mayor en las zonas más urbanizadas y que aquellos universitarios que conviven con otros presentan más Aceptación social, lo que podría deberse al componente intercultural más elevado que caracteriza dichas zonas. Vivir en zonas rurales estuvo relacionado con una mayor Actualización y Coherencia social por la mayor facilidad de entender su dinámica social, mientras que poseer una mayor edad se asoció a una menor capacidad para entender la dinámica social.

Рara concluir, debe destacarse que los participantes de este estudio han obtenido unas puntuaciones elevadas en bienestar social de forma genérica. Esto puede ser atribuido tanto a las titulaciones de corte humanístico que estudia la muestra universitaria, adhiriéndose todas ellas al área de Ciencias de la Educación. Asimismo, el porcentaje femenino ha sido mayor en la muestra de estudio, el cual ha estado ligado tradicionalmente a mayores niveles de bienestar y Contribución social, pudiendo ejercer una notable influencia en los valores hallados por sus características psicosociales intrínsecas. En definitiva, se muestran diferencias en el bienestar social de los jóvenes universitarios basándose en factores de tipo sociodemográfico, siendo de interés la ejecución de acciones y estrategias que permitan la comprensión de las dinámicas sociales y la Actualización social en aquellos sectores que adolecen un menor bienestar, como son los estudiantes de género masculino que residen en entornos urbanos y con sus familias. 


\section{Referencias}

Aknin, L. B., Barrington-Leigh, C. P., Dunn, E. W., Helliwell, J. F., Burns, J., Biswas-Diener, R., ... y Norton, M. I. (2013). Prosocial spending and well-being: cross-cultural evidence for a psychological universal. Journal of Personality and Social Psychology, 104(4), 635-652. https://www.apa.org/pubs/journals/releases/psp104-4-635.pdf

Blanco, A. y Díaz, D. (2005). El bienestar social: su concepto y medición. Psicothema, 17(4), 582-589. http://www.psicothema.com/psicothema.asp?id=3149

Burke, M., Marlow, C. y Lento, (2010). Social network activity and social well-being. Actas de la 28 Conferencia Internacional "Human Factors In Computing Systems" (pp. 1901-1912).

https://doi.org/10.1145/1753326.1753613

Chacón, R., Zurita, F., Castro, M., Espejo, T., Martínez, A. y Lucena, M. (2017). Análisis descriptivo del consumo de sustancias nocivas en estudiantes universitarios de Granada. Revista Complutense de Educación, 28(3), 33-47. https://doi.org/10.5209/rev RCED.2017.v28.n3.50083

Chavarría, M. P. y Barra, E. (2014). Satisfacción vital en adolescentes: relación con la autoeficacia y el apoyo social percibido. Terapia Psicológica, 32(1), 41-46.

https://doi.org/10.4067/S0718-48082014000100004

Cotton, S., Dollard, M. F. y De Jonge, J. (2002). Stress and student job desing: satisfaction, well-being, and performance in university students. International Journal of Stress Management, 9(3), 147-162.

Cookingham, L. M. y Ryan, G. L. (2015). The impact of social media on the sexual and social wellness of adolescents. Journal of Pediatric and Adolescent Gynecology, 28(1), 2-5.

https://doi.org/10.1016/j.jpag.2014.03.001

Diener, E., Wirtz, D., Tov, W., Kim-Prieto, C., Choi, D., Oishi, S. y Biswas-Diener, R. (2010). New well-being measures: short scales to assess flourishing and positive and negative feelings. Social Indicators Research, 97(2), 143-156. https://doi.org/10.1007/s11205-009-9493-y

Ding, Q., Zhang, Y. X., Wei, H., Huang, F. y Zhou, Z. K. (2017). Passive social network site use and subjective well-being among Chinese university students: a moderated mediation model of envy and gender. Personality and Individual Differences, 13, 142-146. https://doi.org/10.1016/i.paid.2017.03.027

Fernández, L. y Gutiérrez, M. (2013). Bienestar social, económico y ambiental para las presentes y futuras generaciones. Información Tecnológica, 24(2), 121-130.

http://dx.doi.org/10.4067/S0718-07642013000200013

Friedman, E. (2011). Sleep quality, social well-being, gender, and inflammation: an integrative analysis in a national sample. Annals of the New York Academy of Sciences, 1231(2011), 23-34.

https://doi.org/10.1111/j.1749-6632.2011.06040.x

González-Peiteado, M., Pino-Juste, M. y Penado, M. (2017). Estudio de la satisfacción percibida por los estudiantes de la UNED con su vida universitaria. Revista Iberoamericana de Educación a Distancia, 20(1), 243-260. http://revistas.uned.es/index.php/ried/article/view/16377

Haapanen, M. y Tervo, H. (2012). Migration of the highly educated: evidence from residence spell of university graduates. Journal of Regional Science, 52(4), 587-605. https://doi.org/10.1111/1.1467$\underline{9787.2011 .00745 . x}$ 
Keyes, C. y Shapiro, A. (1998). Social well-being in the United States: a descriptive epidemiology. En O. G. Brim, C. Ryff y R. Kessler (Eds.), How healthy are we? (pp. 350-372). University of Chicago Press.

Lalive, R. y Stutzer, A. (2010). Approval of equal rights and gender differences in well-being. Journal of Population Economics, 23(3), 933-962. https://doi.org/10.1007/s00148-009-0257-4

López, R. N. y Wodtke, G. (2010). College residence and academic performance: who benefits from living on campus? Urban Education, 45(4), 506-532. https://doi.org/10.1177/0042085910372351

Lorenzo, M., Argos, J., Hernández, J. y Vera, J. (2014). El acceso y la entrada del estudiante a la universidad: situación y propuestas de mejora facilitadoras del tránsito. Educación XXI, 17(1), 15-38.

https://doi.org/10.5944/educxx1.17.1.9951

Luong, G., Charles, S. T. y Fingerman, K. (2011). Better with age: social relationships across adulthood. Journal of Social and Personal Reltionships, 28(1), 9-23. https://doi.org/10.1177/0265407510391362

Martínez-Martínez, A., Zurita-Ortega, F., Castro-Sánchez, M., Chacón-Cuberos, R., Hinojo-Lucena, M. A. y Espejo-Garcés, T. (2016). La elección de estudio superiores universitarios en estudiantes de último curso de bachillerato y ciclos formativos. Revista Electrónica Educare, 20(1), 1-18.

https://doi.org/10.15359/ree.20-1.14

Oberle, E., Schonert-Reichl, K. A. y Thomson, K. C. (2010). Understanding the link between social and emotional well-being and peer relations in early adolescence: gender-specific predictors of peer acceptance. Journal of Youth Adolescence, 39, 1330-1342. https://doi.org/10.1007/s10964-009-9486-9

Oishi, S. (2010). The Psychology of residential mobility: implications for the self, social relationships, and well-being. Perspectives on Psychological Science, 5(1), 5-21. https://doi.org/10.1177/1745691609356781

Oswald, F., Jopp, D., Rott, C. y Wahl, H. W. (2011). Is aging in place a resource for or risk to life satisfaction? The Gerontologist, 51(2), 238-250. https://doi.org/10.1093/geront/gna096

Perry-Parrish, C. y Zeman, J. (2011). Relations among sadness regulation, peer acceptance, and social functioning in early adolescence: the role of gender. Social Development, 20(1), 135-153.

https://doi.org/10.1111/j.1467-9507.2009.00568.x

Rollero, C. y De Piccoli, N. (2010). Does place attachment affect social well-being? Revue Européenne de Psychologie Appliquée, 60(2010), 233-238. https://doi.orq/10.1016/i.erap.2010.05.001

Salanova, M., Cifre, E., Grau, R., Llorens, S. y Martínez, I. M. (2005). Antecedentes de la autoeficacia en profesores y estudiantes universitarios: un modelo causal. Revista de Psicología del Trabajo y de las Organizaciones, 21(1), 159.176.

Yu, S., Kerres, C. y Kilpatrick, M. (2010). Relationship between multiple sources of perceived social support and psychological and academic adjustment in early adolescence: comparisons across gender. Journal of Youth Adolescence, 39, 47-61. https://doi.org/10.1007/s10964-008-9368-6

Zubieta, E. y Delfino, G. (2010). Satisfacción con la vida, bienestar psicológico y bienestar social en estudiantes universitarios de buenos aires. Anuario de Investigaciones, 17, 277-283. 